\section{International Scientific Journal Theoretical \& Applied Science}

p-ISSN: 2308-4944 (print) e-ISSN: 2409-0085 (online)

Year: 2015 Issue: 05 Volume: 25

Published: $30.05 .2015 \quad$ http://T-Science.org
SECTION 4. Computer science, computer engineering and automation.
Alexandr Petrovich Kirpichnikov

Dr. Sci, Prof,

Head of the Department of Intelligent Systems \&

Information Systems Control, KNRTU, Russia kirpichnikov@kstu.ru

Dmitry Borisovich Flax

Senior Lecturer of the Department of Automated Data Acquisition \& Processing Systems, KNRTU, Russia flaxdm@gmail.com

Liliya Rustamovna Valeeva master of study of the Department of Automated Data Acquisition \& Processing Systems, KNRTU, Russia lilya.cat20@gmail.com

\title{
PROBABILISTIC CHARACTERISTICS OF OPEN MULTI-CHANNEL QUEUING SYSTEM WITH LIMITED AVERAGE RESIDENCE TIME OF THE APPLICATION IN THE SYSTEM
}

\author{
Abstract: The mathematical model of multi-channel queuing system of open type with bounded mean residence \\ time of the application in the system is presented. A detailed mathematical formalization of the model is held; the \\ probabilistic characteristics of queuing system of this type are calculated. \\ Key words: queuing system, flow of requirements, queue, serving device. \\ Language: Russian \\ Citation: Kirpichnikov AP, Flax DB, Valeeva LR (2015) PROBABILISTIC CHARACTERISTICS OF OPEN \\ MULTI-CHANNEL QUEUING SYSTEM WITH LIMITED AVERAGE RESIDENCE TIME OF THE \\ APPLICATION IN THE SYSTEM. ISJ Theoretical \& Applied Science 05 (25): 44-49. \\ Soi: http://s-o-i.org/1.1/TAS*05(25)9 Doi: crossef http://dx.doi.org/10.15863/TAS.2015.05.25.9
}

УДК 519.872

\section{ВЕРОЯТНОСТНЫЕ ХАРАКТЕРИСТИКИ ОТКРЫТОЙ МНОГОКАНАЛЬНОЙ СИСТЕМЫ МАССОВОГО ОБСЛУЖИВАНИЯ С ОГРАНИЧЕННЫМ СРЕДНИМ ВРЕМЕНЕМ ПРЕБЫВАНИЕ ЗАЯВКИ В СИСТЕМЕ}

Аннотация: Представлена математическая модель открытой многоканальной системы массового обслуживания с ограниченным средним временем пребывания заявки в системы. Проведена подробная математическая формализация модели и вычислены вероятностные характеристик систем массового обслуживания этого типа.

Ключевые слова: система массового обслуживания, поток требований, очередь, обслуживающее устройство.

В настоящее время значительный интерес представляет исследование таких систем массового обслуживания, для которых общее время пребывания одной заявки в системе в целом (как в очереди, так и под обслуживанием) ограничено некоторым случайным временем $t$ со средним значением $\bar{t}$. С такого рода системами массового обслуживания приходится иметь дело достаточно часто. Например, при противовоздушной обороне воздушная цель может пробыть в зоне стрельбы (зоны действия обслуживающего устройства) лишь некоторое ограниченное время и покидает её независимо от того, была ли она обслужена (то есть, кончился обстрел или нет). Другим примером таких систем являются приборы (например, счётчика элементарных частиц), область действия которых ограничено некоторой зоной действия, вне которой прибор не работает. При этом каждое требование, проходящее через эту зону, может быть обслужено прибором, если он не занят, но за пределами зоны обслуживания прибор уже лишён возможности обслуживать требования. В данной работе предложен вариант комплексного описания систем массового обслуживания с ограниченным средним временем ожидания заявок в системе на основе того математического аппарата, который был ранее представлен и развит авторами в работе [1]. Напомним, что в этой работе была подробно изучена 
одноканальная система массового обслуживания (СМО) с ограниченным средним временем ожидания заявок в очереди. Математической основой при этом является введение в рассмотрение функции Миттаг-Леффлера первого порядка $E_{1}(z ; \mu)$ [2, 3], определяемой формулой

$$
E_{1}(z ; \mu)=\sum_{k=0}^{\infty} \frac{z^{k}}{\Gamma(\mu+k)},
$$

где $Г-$ гамма-функция, позволяющей значительно упростить большинство промежуточных расчётов. Полученная на этой основе единая, внутренне связанная, система сравнительно компактных формул позволила адекватно описать все основные характеристики стационарных режимов такого рода одноканальной СМО - вероятность простоя системы $p_{0}$, коэффициента загрузки $\bar{m}$ и среднюю длину очереди $\bar{l}$, а также вычислить соответствующие этим характеристикам временные величины. Для изучения многоканальной системы массового обслуживания такого типа, однако, использованный в работе [1] математический аппарат следует скорректировать с учётом тех задач, которые ставит перед исследователем изучение системы массового обслуживания с числом каналов большим, чем единица.

Предположим, что мы имеем многоканальную СМО с однородным бесконечным простейшим потоком заявок и очередью неограниченной длины. Пусть интенсивность потока заявок равна $\lambda$, а интенсивность обслуживания, то есть среднее число заявок, которые обслуживает прибор в единицу времени, есть $\mu$. Поток обслуживания тоже будем считать простейшим (с интенсивностью $\mu$ ).

Предположим далее, что общее время пребывания одной заявки в системе ограничено теперь некоторым случайным временем $t$ со средним значением $\bar{t}$. Тем самым, на каждую заявку, находящуюся в системе, действует поток уходов с интенсивностью

$$
\mathrm{v}=\frac{1}{\bar{t}} .
$$

Ясно, что если этот поток носит простейший характер, то процесс, протекающий в СМО, будет марковским. Найдём для него вероятности стационарных состояний. Если в системе находится $S$ заявок, то при этом суммарная интенсивность уходов заявок из системы равна $s \mathrm{~V}$, и тогда граф состояний такого рода системы массового обслуживания имеет вид, изображённый на рис. 1:

Как видно из графа, перед нами классическая схема процесса гибели и размножения. Применив общие выражения (например, $[4,5])$ для вероятностей предельных (стационарных) состояний в этой схеме, получим

$$
\begin{aligned}
& p_{1}=\frac{\lambda}{\mu+v} p_{0} ; p_{2}=\frac{\lambda^{2}}{2(\mu+v)^{2}} p_{0} ; \\
& p_{3}=\frac{\lambda^{3}}{3 !(\mu+v)^{3}} p_{0} \cdots p_{m}=\frac{\lambda^{m}}{m !(\mu+v)^{m}} p_{0} ; \\
& p_{m+1}=\frac{\lambda^{m+1} p_{0}}{m !(\mu+v)^{m}[m(\mu+v)+v]} ;
\end{aligned}
$$

будет марковским. Найдём для него вероятности стационарных состояний. Если в системе находится $S$ заявок, то при этом суммарная интенсивность будет марковским. Найдём для него вероятности стационарных состояний. Если в системе находится $S$ заявок, то при этом суммарная интенсивность

$$
\begin{gathered}
p_{m+2}= \\
=\frac{\lambda^{m+2} p_{0}}{m !(\mu+v)^{m}[m(\mu+v)+v][m(\mu+v)+2 v]}
\end{gathered}
$$

$$
\begin{gathered}
p_{m+3}= \\
=\frac{\lambda^{m+3} p_{0}}{m !(\mu+v)^{m}[m(\mu+v)+v][m(\mu+v)+2 v][m(\mu+v)+3 v]}
\end{gathered}
$$$$
\cdots \text {. }
$$ 

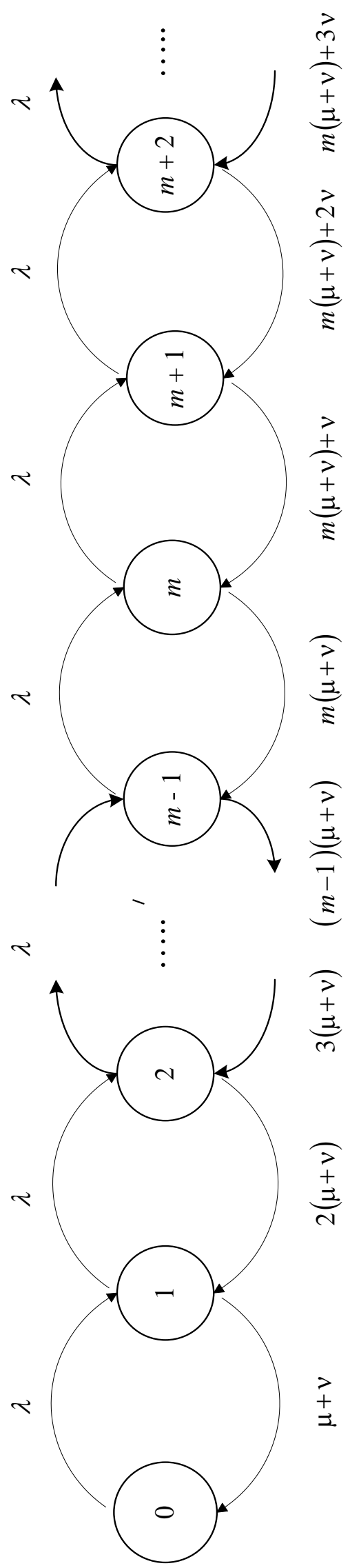

Рисунок 1 - Граф состояний системы массового обслуживания. 
Введём далее обозначение $\tilde{\mu}=\mu+v$, тогда, очевидно, имеем

$$
\begin{gathered}
p_{1}=\frac{\lambda}{\tilde{\mu}} p_{0} ; p_{2}=\frac{\lambda^{2}}{2 \tilde{\mu}^{2}} p_{0} ; p_{3}=\frac{\lambda^{3}}{3 ! \tilde{\mu}^{3}} p_{0} \cdots p_{m}=\frac{\lambda^{m}}{m ! \tilde{\mu}^{m}} p_{0} ; \\
p_{m+1}=\frac{\lambda^{m+1}}{m ! \tilde{\mu}^{m}(m \tilde{\mu}+v)} p_{0} ; p_{m+2}=\frac{\lambda^{m+2}}{m ! \tilde{\mu}^{m}(m \tilde{\mu}+v)(m \tilde{\mu}+2 v)} p_{0} ; \\
p_{m+3}=\frac{\lambda^{m+3} p_{0}}{m ! \mu^{m}(m \tilde{\mu}+v)(m \tilde{\mu}+2 v)(m \tilde{\mu}+3 v)} \cdots
\end{gathered}
$$

или

$$
\begin{gathered}
p_{1}=\tilde{\rho} p_{0} ; p_{2}=\frac{\tilde{\rho}^{2}}{2} p_{0} ; p_{3}=\frac{\tilde{\rho}^{3}}{3 !} p_{0} ; \cdots p_{m}=\frac{\tilde{\rho}^{m}}{m !} p_{0} ; p_{m+1}=\frac{\tilde{\rho}^{m+1}}{m !(m+\tilde{\beta})} p_{0} \\
p_{m+2}=\frac{\tilde{\rho}^{m+2}}{m !(m+\tilde{\beta})(m+2 \tilde{\beta})} p_{0} ; p_{m+3}=\frac{\tilde{\rho}^{m+3}}{m !(m+\tilde{\beta})(m+2 \tilde{\beta})(m+3 \tilde{\beta})} p_{0} \cdots
\end{gathered}
$$

где $\quad \tilde{\rho}=\frac{\lambda}{\tilde{\mu}}=\frac{\lambda}{\mu+v}=\frac{\rho}{1+\beta}$,

$\tilde{\beta}=\frac{v}{\tilde{\mu}}=\frac{v}{\mu+v}=\frac{\beta}{1+\beta}$.

При этом $\rho=\frac{\lambda}{\mu} \quad-\quad$ приведённая интенсивность потока заявок в систему, а $\beta=\frac{\nu}{\mu}$ приведённая интенсивность ухода «нетерпеливых» заявок из системы. Напомним, что параметры $\rho$ и $\beta$ показывают соответственно, сколько в среднем заявок поступает в систему и покидает её необслуженными за среднее время обслуживания системой одной заявки. В общем виде, очевидно, имеем следующие формулы для $p_{k}$ :

$$
\begin{gathered}
p_{k}=\frac{\tilde{\rho}^{k}}{k !} p_{0} \quad \text { при } k \leq m ; \\
p_{k}=\frac{\tilde{\rho}^{k}}{m !(m+\tilde{\beta})(m+2 \widetilde{\beta}) \cdots[m+(k-m) \tilde{\beta}]^{p_{0}}} \\
\text { при } k>m .
\end{gathered}
$$

Запись формул (1) для $p_{k}$ можно упростить следующим образом. Разделим числитель и знаменатель второго из этих соотношений на $\tilde{\beta}^{k-m}$. Тогда получим

$$
p_{k}=\frac{\tilde{\rho}^{k}}{k !} p_{0} \quad \text { при } k \leq m ;
$$

$$
p_{k}=\frac{\tilde{\rho}^{m}}{m !} \frac{\alpha^{k-m}}{(m / \tilde{\beta}+1)_{k-m}} p_{0} \quad \text { при } k \geq m \text {. }
$$

В этом соотношении

$(a)_{k}=a(a+1)(a+2) \ldots(a+k-1)$;

$(a)_{0}=1$ - символ Похгаммера [например, 6], при этом $(1)_{k}=k$ !. Величина $\alpha=\tilde{\rho} / \tilde{\beta}=\lambda / v$, очевидно, показывает, какое среднее число заявок поступает в систему за среднее время пребывания в очереди одной «нетерпеливой» заявки. В этом случае из условия нормировки имеем

$$
\begin{gathered}
p_{0}=\left\{e_{m-1}(\tilde{\rho})+\frac{\tilde{\rho}^{m}}{m !}\left[1+\frac{\alpha}{m / \tilde{\beta}+1}+\right.\right. \\
\left.\left.+\frac{\alpha^{2}}{(m / \tilde{\beta}+1)(m / \tilde{\beta}+2)}+\frac{\alpha^{3}}{(m / \tilde{\beta}+1)(m / \tilde{\beta}+2)(m / \tilde{\beta}+3)}+\cdots\right]\right\}^{-1}=
\end{gathered}
$$




$$
\begin{gathered}
=\left\{e_{m-1}\{\tilde{\rho}\}+\frac{\tilde{\rho}^{m}}{m !}\left[1+\frac{\alpha}{(m / \tilde{\beta}+1)_{1}}+\frac{\alpha^{2}}{(m / \tilde{\beta}+1)_{2}}+\frac{\varepsilon^{3}}{(m / \tilde{\beta}+1)_{3}}+\cdots\right]\right\}^{-1}= \\
=\left[e_{m-1}(\tilde{\rho})^{+}+\frac{\tilde{\rho}^{m}}{m !} \sum_{k=0}^{\infty} \frac{\alpha^{k}}{(m / \tilde{\beta}+1)_{k}}\right]^{-1} \cdot
\end{gathered}
$$

В этом соотношении

$$
e_{m}(\tilde{\rho})=1+\frac{\tilde{\rho}}{1 !}+\frac{\tilde{\rho}^{2}}{2 !}+\ldots+\frac{\tilde{\rho}^{m}}{m !}
$$

- неполная экспоненциальная функция (неполная экспонента). При этом $e_{0}(\tilde{\rho})=1$, а при $m<0$ полагаем $e_{m}(\tilde{\rho})=0$. Ясно, что $e_{m}(\tilde{\rho}) \rightarrow e^{\tilde{\rho}}$ при $m \rightarrow \infty$.

Рассмотрим более внимательно сумму в формуле (1). Ясно, что в отличие от соответствующего соотношения классической модели $\mathrm{M} / \mathrm{M} / \mathrm{m}$, в котором сумма бесконечного числа слагаемых в знаменателе этой формулы сводились к сумме бесконечной геометрической прогрессии, в формуле (1) содержится сумма $S$ бесконечного ряда, не являющегося такого рода прогрессией. Поэтому будем действовать следующим образом.

Введём в рассмотрение вырожденную гипергеометрическую функцию Куммера ${ }_{1} F_{1}(a ; b ; z)$, определяемую формулой [7]

$$
{ }_{1} F_{1}(a ; b ; z)=\sum_{k=0}^{\infty} \frac{(a)_{k}}{(b)_{k}} \frac{z^{k}}{k !}
$$

в этом случае, очевидно, имеем

$$
S=\sum_{k=0}^{\infty} \frac{\alpha^{k}}{(m / \widetilde{\beta}+1)_{k}}={ }_{1} F_{1}(1 ; m / \widetilde{\beta}+1 ; \alpha)
$$

поскольку $(1)_{k}=k$ !. Отсюда получаем более компактную формулу для вероятности полного простоя системы $p_{0}$ :

$$
p_{0}=\left[e_{m-1}(\tilde{\rho})+\frac{\tilde{\rho}^{m}}{m !}{ }_{1} F_{1}(1 ; m / \tilde{\beta}+1 ; \alpha)\right]^{-1} .
$$

Последнее выражение, в свою очередь, можно еще больше упростить следующим образом. Замечая, что per definitio

$$
(a)_{k}=\frac{\Gamma(a+k)}{\Gamma(a)},
$$

где Г - гамма-функция, перепишем выражение для вырожденной гипергеометрической функции как

$$
{ }_{1} F_{1}(a ; b ; z)=\frac{\Gamma(b)}{\Gamma(a)} \sum_{k=0}^{\infty} \frac{\Gamma(a+k)}{\Gamma(b+k)} \frac{z^{k}}{k !}
$$

а интересующую нас сумму как

$$
S={ }_{1} F_{1}(1 ; \xi+1 ; z)=\Gamma(\xi+1) \sum_{k=0}^{\infty} \frac{z^{k}}{\Gamma(\xi+1+k)},(4)
$$

поскольку $\Gamma(k+1)=k$ !. Далее для упрощения последнего выражения используем следующую цепочку формул:

$$
\begin{gathered}
\sum_{k=0}^{\infty} \frac{z^{k}}{\Gamma(\xi+1+k)}=\sum_{k=1}^{\infty} \frac{z^{k-1}}{\Gamma(\xi+k)}=\frac{1}{z} \sum_{k=1}^{\infty} \frac{z^{k}}{\Gamma(\xi+k)}= \\
=\frac{1}{z}\left[\sum_{k=0}^{\infty} \frac{z^{k}}{\Gamma(\xi+k)}-\frac{1}{\Gamma(\xi)}\right] .
\end{gathered}
$$

Таким образом, получаем

$$
S={ }_{1} F_{1}(1 ; \xi+1 ; z)=\frac{\Gamma(\xi+1)}{z}\left[\sum_{k=0}^{\infty} \frac{z^{k}}{\Gamma(\xi+k)}-\frac{1}{\Gamma(\xi)}\right],
$$


откуда с учетом известного рекуррентного соотношения для гамма-функции [7, 8] $\Gamma(\xi+1)=\xi \Gamma(\xi)$ следует

$$
\begin{gathered}
S={ }_{1} F_{1}(1 ; \xi+1 ; z)= \\
=\frac{\xi}{z}\left[\Gamma(\xi) \sum_{k=0}^{\infty} \frac{\alpha^{k}}{\Gamma(\xi+k)}-1\right]=\frac{\xi}{z}\left[\sum_{k=0}^{\infty} \frac{я^{k}}{(\xi)_{k}}-1\right]
\end{gathered}
$$

или

$$
S={ }_{1} F_{1}(1 ; \xi+1 ; z)=\frac{\xi}{z}\left[F_{1}(1 ; \xi ; z)-1\right]
$$

- рекуррентная формула для ${ }_{1} F_{1}(1 ; \xi ; z)$.

В данном случае, очевидно, имеем $\xi=m / \tilde{\beta}, \quad z=\alpha=\tilde{\rho} / \tilde{\beta}, \quad$ в этом случае $\xi / z=m / \tilde{\rho}$ и тогда, применяя формулу (5) к соотношению (3), получаем следующую компактную формулу для вероятности полного простоя системы $p_{0}$ :

$$
p_{0}=\left[e_{m-2}(\rho)+\frac{\rho^{m-1}}{(m-1) !}{ }_{1} F_{1}(1 ; m / \tilde{\beta} ; \alpha)\right]^{-1}
$$

(напомним, что $e_{0}(\rho)=1$ и $e_{m}(\rho)=0$ для всех $m<0)$. Для одноканальной $\mathrm{CMO} \quad(m=1)$ последнее выражение имеет особенно простой вид

$$
p_{0}=\frac{1}{{ }_{1} F_{1}(1 ; 1 / \tilde{\beta} ; \alpha)}
$$

Легко также показать, что при $\tilde{\beta} \rightarrow 0$

$$
\left.\lim _{1} F_{1}(1 ; 1 / \tilde{\beta} ; \alpha)\right|_{\tilde{\beta} \rightarrow 0}=\frac{1}{1-\rho},
$$

и тогда в пределе $\tilde{\beta} \rightarrow 0, \quad$ который соответствует случаю классической СМО (по классификации Кендалла - модель M/M/1) формула (6) переходит в известное соотношение $p_{0}=1-\rho$ классической модели.

\section{References:}

1. Busarev MI, Kirpichnikov AP, Flaks DB (2011) Vestnik Kazan. tekhnol. un-ta, 5, 22, pp. 155161 (2011).

2. Dzhrbashyan MM (1966) Integral'nye preobrazovaniya i predstavleniya funktsiy. Moscow, Nauka, 1966. 672 p.

3. Gol'dberg AA, Ostrovskiy IV (1970) Raspredelenie znacheniy meromorfnykh funktsiy. Moscow, Nauka, 1970. 591 p.

4. Venttsel ES (1972) Issledovanie operatsiy. Moscow, Sovetskoe radio, 1972. 552 p.
5. Kirpichnikov AP (2011) Metody prikladnoy teorii massovogo obsluzhivaniya. Kazan', Izdvo Kazanskogo universiteta, 2011. 200 p.

6. Prudnikov AP, Brychkov YA, Marichev OI (1983) Integraly i ryady. Spetsial'nye funktsii. Nauka, Moscow, 1983. 664 p.

7. Prudnikov AP, Brychkov YA, Marichev OI (1985) Integraly i ryady. Dopolnitel'nye glavy. Moscow, Nauka, 1985. 800 p.

8. Abramovits M, Stigan I (1977) Spravochnik po spetsial'nym funktsiyam. Nauka, Moscow, 1977. $832 \mathrm{p}$. 